\title{
ADVO: A System to Manage Influencer Marketing Campaigns on Social Networks
}

\author{
Tai Huynh ${ }^{1,2, \dagger}$, Hien D. Nguyen ${ }^{3,4, *,+}$, Ivan Zelinka ${ }^{5,6}$, Kha V. Nguyen ${ }^{2}$, Vuong T. Pham ${ }^{7}$ and Suong N. Hoang ${ }^{8}$ \\ 1 Faculty of Information Technology, Ton Duc Thang University, Ho Chi Minh City 700000, Vietnam; \\ tai.huynh@kyanon.digital \\ 2 Kyanon Digital, Ho Chi Minh City 700000, Vietnam; kha.nguyen@kyanon.digital \\ 3 Faculty of Computer Science, University of Information Technology, Ho Chi Minh City 700000, Vietnam \\ 4 Vietnam National University, Ho Chi Minh City (VNU-HCM), Quarter 6, Thu Duc District, \\ Ho Chi Minh City 700000, Vietnam \\ 5 Modeling Evolutionary Algorithms Simulation and Artificial Intelligence, Faculty of Electrical and Electronics \\ Engineering, Ton Duc Thang University, Ho Chi Minh City 700000, Vietnam; ivan.zelinka@tdtu.edu.vn \\ 6 Department of Computer Sciences, FEI VBS Technical University of Ostrava, Tr. 17. Listopadu 15, \\ 70800 Ostrava, Czech Republic \\ 7 Faculty of Information Technology, Sai Gon University, Ho Chi Minh City 700000, Vietnam; \\ vuong.pham@sgu.edu.vn \\ 8 Olli Technology, Ho Chi Minh City 700000, Vietnam; suong@olli-ai.com \\ * Correspondence: hiennd@uit.edu.vn \\ + Equal contribution by Tai Huynh and Hien D. Nguyen.
}

Citation: Huynh, T.; Nguyen, H.D.; Zelinka, I.; Nguyen, K.V.; Pham, V.T.; Hoang, S.N. ADVO: A System to

Manage Influencer Marketing Campaigns on Social Networks. Appl. Sci. 2021, 11, 6497. https://doi.org/ 10.3390/app11146497

Academic Editors: Paola Velardi and Stefano Faralli

Received: 3 June 2021

Accepted: 7 July 2021

Published: 15 July 2021

Publisher's Note: MDPI stays neutral with regard to jurisdictional claims in published maps and institutional affiliations.

Copyright: (c) 2021 by the authors. Licensee MDPI, Basel, Switzerland. This article is an open access article distributed under the terms and conditions of the Creative Commons Attribution (CC BY) license (https:// creativecommons.org/licenses/by/ $4.0 /)$.
Featured Application: This paper presents a method to design a system to manage influencer marketing campaigns. The designed system can determine emerging influencers for a brand by using these measures about amplification factors for evaluating information propagation, the passion point to measure the favorite of a user of a brand, and the content creation score for determining the ability of post-content creation. In a marketing campaign, the designing system manages the parameters of emerging influencers, such as the information diffusion of each influencer, and determines statistically their effectiveness by presenting the conversion rate of clicks and orders, the number of clicks on interactions, and their revenue. Moreover, this system also supports the customer's decision at that moment based on producing the data visualization of the campaign's results. Therefore, the designed management system is helpful for application in an influencer marketing campaign in practice.

Abstract: In the fourth technology revolution, influencer marketing is an essential kind of digital marketing. This marketing uses identified influencers to viral the information of products to target customers. It is useful to support brands exposed to more valuable online consumers. The influencer marketing campaign needs a management system to manage on a social network. This system helps to increase the efficiency of a campaign. This paper proposes a management system for the influencer marketing campaign, called the ADVO system. This system provides a tool for collecting data on a social network and detecting potential brand influencers for the marketing campaign. The meaningful measures for users include amplification factors for evaluating the information propagation, the passion point to measure a user's favorite when it comes to a brand, and the content creation score for determining the ability of post-content creating. The ADVO system helps the brand to make the decision through real-time visual reports of the campaign. It is a foundation to create commercial activities and construct an advocate community of the related brand.

Keywords: content creation; opinion leaders; social network; brand advocate; influencer marketing; digital marketing; Facebook 


\section{Introduction}

Digital marketing uses the internet, social media, mobile applications, and other digital channels to reach consumers [1]. Influencer marketing is an important kind of digital marketing [1,2]. It uses recommendations and product mentions from influencers-individuals with a purported expert level of knowledge or social influence in their niche [3,4]. The influencer marketing campaign is expected to viral the brand through their personal social channels [5]. This helps to diffuse its information to the larger market, mainly targeted customers [6].

An influencer marketing campaign will work effectively if the influencers of that campaign match the target audience of the brand and the goals. The valuable influencers help the brand build trust through authentic endorsements and propagate the brand's information to potential consumers [7]. Through that, this impacts consumer buying decisions. Hence, determining suitable influencers is important. Marketers have three ways to find influencers on social media: searching on social networks, platforms of influencers or some influencers reached to them before [5,8].

When doing an influencer marketing campaign, the brand requires a system to manage the campaign's work. Using the information of the brand and consumers, this management system can crawl data on the social network. The system has equipped a model to organize collected data that represents the relations between social network objects. Besides, the data organization model can also help the brand detect emerging influencers that can be used for running its influencer marketing campaign. Detecting influencers is based on determining some measures of computing the influence of users on their audience. Finally, the management system carries the brand to give the decision. For decision-supporting, it traces the traffic from each influencer to estimate customers' quality and shows visual reports to summarize the campaign results in real-time. Some valuable parameters evaluate the marketing campaign's effectiveness: the interactions, conversation rate per click, and revenue of each influencer.

This study proposes a method to design a management system used in influencer marketing campaigns on a social network. This system is called the ADVO system. Besides the management of marketing campaigns, this system is a foundation supporting businesses to establish their e-commercial activities and construct their advocate community. Some influential measures for determining potential influencers are proposed and studied: amplification factors to estimate information propagation in a duration time [9], the passion point to determine the fondness of a user with the brand, and the creation score to evaluate ability in content creation (Figure 1).

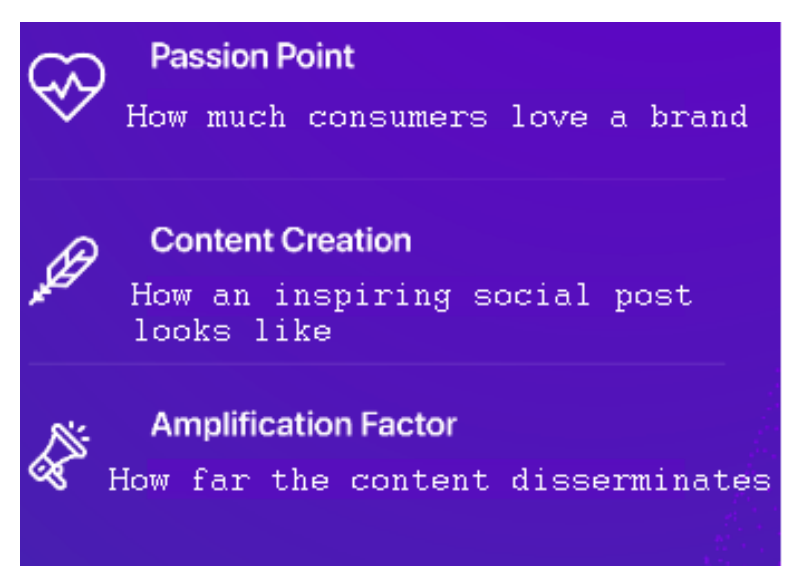

Figure 1. The influential measures of the ADVO system.

There are three main sub-systems in the ADVO system: AdvoSights—-this system works for social listening and analyzing behaviors of users on a social network, AdvoBiz-the 
foundation that manages commercial activities of a brand, and AdvoFair-the foundation that helps the brand build and optimize values of the brand's advocate community.

Some related works about the management of influencers marketing campaigns are presented in Section 2. There are some studies on detecting influential users on the social network. The following section describes the architecture of the ADVO system and its work. This management system has three main sub-systems: AdvoSights, AdvoBiz, and AdvoFair. Section 4 constructs some influential measures to detect potential influencers for a determined brand. The experimental results are shown in Section 5 when the ADVO system is used for a real-world influencer marketing campaign in fashion. The conclusion of this paper and some future works are presented in the last section.

\section{Related Works}

The social network is a convenient foundation to extract human activities $[10,11]$. There are many methods for determining influencers on a social network [12]. Current methods are designed based on information propagation with relations between users and others [13-15]. Some factors impacting characteristics of the content propagation are media type, content type, the level of engagement through the number of reactions, comments, and shares at brand pages on social networks such as Facebook and Twitter $[16,17]$. Those methods have not reached some characteristics of a brand, such as a user's love of a brand, the inspiration of the content of a user's posts, or estimating the total of interactions in a duration time.

Riquelme et al. built influent measures for a user on Twitter [18]. Those measures include almost all values about the affection of users to their audience on Twitter. However, they have not yet studied the diffusion of tweets on a network. The study in [19] proposed a method to evaluate the propagation speed of the post in the duration time, but it does not still show full information of an influencer on a network.

Using the nomological network to represent relations between brands and their customers, Laroche et al. recommended some parameters for computing the customers' loyalty with brands through social media [8]. Nonetheless, that study does not mention estimating the inspiration of post content related to the brand.

Tidke et al. proposed two methods, average consensus ranking aggregation (ACRA) and weighted average consensus ranking aggregation (WACRA), by using heterogeneous surface learning features [20]. Those methods are used to determine nodes influencing social networks. They also experimented on practical data, which were collected from Twitter for economic and political topics. Nevertheless, the results of those methods do not associate with the brand feature, and they are not valuable for influencer marketing of the responding brand.

In [21], the authors presented the K-path algorithm to select influential nodes to optimize the influence spread on large-scale social networks. However, this method does not apply to compute the passion of a user with a brand. Hence, it not effective when detecting emerging influencers for a market campaign of the brand.

Taheri and Bouyer proposed the improved version of affinity propagation with an adaptive similarity matrix to detect communities in social networks [22]. However, it cannot detect communities interested in a brand based on the loving of members in the group with that brand.

Moreover, the current systems for carrying out influencer marketing have not yet met business demands completely. Based on users who join those systems, they can search influencers for a determined brand. They cannot manage the process while running a campaign sufficiently.

For example, some systems support the searching of relations between a brand and its set of determined influencers [23-25]. The parameters of those systems for evaluating the influence of users are amplification factors, such as the number of followers, friends, and the number of interactions with them. In [26], the attraction of a post was computed by integrating the passion of users and their amplification factors. The estimation of the post's 
attraction helps to realize the reactions to the post [27]. However, those methods did not mention the combination between a user's fondness for a brand and the ability to create content of posts to identify emerging influencers for an influencer marketing campaign of the corresponding brand.

The SCWord system gives an overview of the relationship between topic clusters on social networks by tracking sentiment changes in real-time [28]. It also displays charts performing the changes in attitudes of users on the social media network. It summarizes the aggregate polarization of posts. Nevertheless, this system cannot be used to detect influencers for a marketing campaign.

The current management systems only determine the key opinion leaders (KOL) and celebrities; they cannot search minor influencers. Hence, the benefit of the campaign is not optimized. Besides, those systems were not supported to manage the evaluation values of an influencer marketing campaign in real-time.

ADVO system is an authenticity-based social commerce platform that motivates social media users to influence their community with authentic and inspiring content. This system is an optimal solution to manage influencer marketing campaigns with full functions.

\section{Design of the Management System for an Influencer Marketing Campaign}

This kind of management system has to be able to control the factors of a marketing campaign. Firstly, this system stores the necessary data to support the brands' greater understanding of their market, especially targeted customers. Secondly, it can help a brand recognize some valuable influencers who can impact their audiences about the information of related products. It analyzes the information on a social network to measure the affection of users for their audiences. Thirdly, the system can visualize real-time statistical data in a campaign, which helps the brand make a reasonable decision. At last, it is the foundation for evaluating the effectiveness of influencer marketing campaigns and building an advocate community for the brand. In this section, the ADVO system, which is a management system for influencer marketing, and its architecture are proposed.

\subsection{The Architecture of the ADVO System}

Based on some determined parameters of a consumer, the ADVO system can be set up to crawl social media data in the duration time automatically. The crawled data is pre-processed to organize as a knowledge base of the consumer. Using the information of data, the system quantifies the influent measures of users to determine potential influencers for that consumer. Those measures are mentioned in $[19,26]$, which are: the amplification factors for evaluating the information propagation in a duration time, the passion point for measuring the favorite with a brand or a consumer, and the content creation score for estimating the ability to create the content on a social network [27]. Moreover, the ADVO system can follow all interactions with the prospective influencers and their revenue in the marketing campaign. It can estimate the viral of each influencer and statistically determine the influencers' effectiveness by presenting the number of clicks on interactions, the conversion rate of clicks and orders, and their revenue. Moreover, this system also supports the customer's decision at the moment based on producing the data visualization of the campaign's results in real-time. Figure 2 shows the architecture of the ADVO system. There are three main functions in the advocates of this architecture: AdvoSights, AdvoBiz, and AdvoFair.

AdvoSights: This is a principal function of the ADVO system. It is a tool to analyze the information of users on a social network. It is also used for social listening to measure the influence of users. It computes some values to detect potential influencers for a brand. Those measures include amplification factors, passion point, and content creation score, estimated in Section 4. They are applied to evaluate and improve the impact of users and the seduction of their audience for interacting with their posts.

AdvoBiz: This is a system supporting businesses to deploy their e-commercial activities. It can collect data from a social media network. It is also a tool to manage campaigns of 
influencer marketing. The brand creates and observes a campaign through AdvoBiz. The system will visualize the campaign report at a time. It summarizes the rate of conversation per click, total interactions, etc., as visual dashboards. Based on that information, the brand can estimate the campaign's benefit and make a decision correctly.

AdvoFair: Brands need to build their advocate community on social networks to viral their new information of products quickly. AdvoFair is a foundation for them to build a homography for the brand. It aims to spread the information of the brand rapidly. The targeted customers will receive product information naturally.

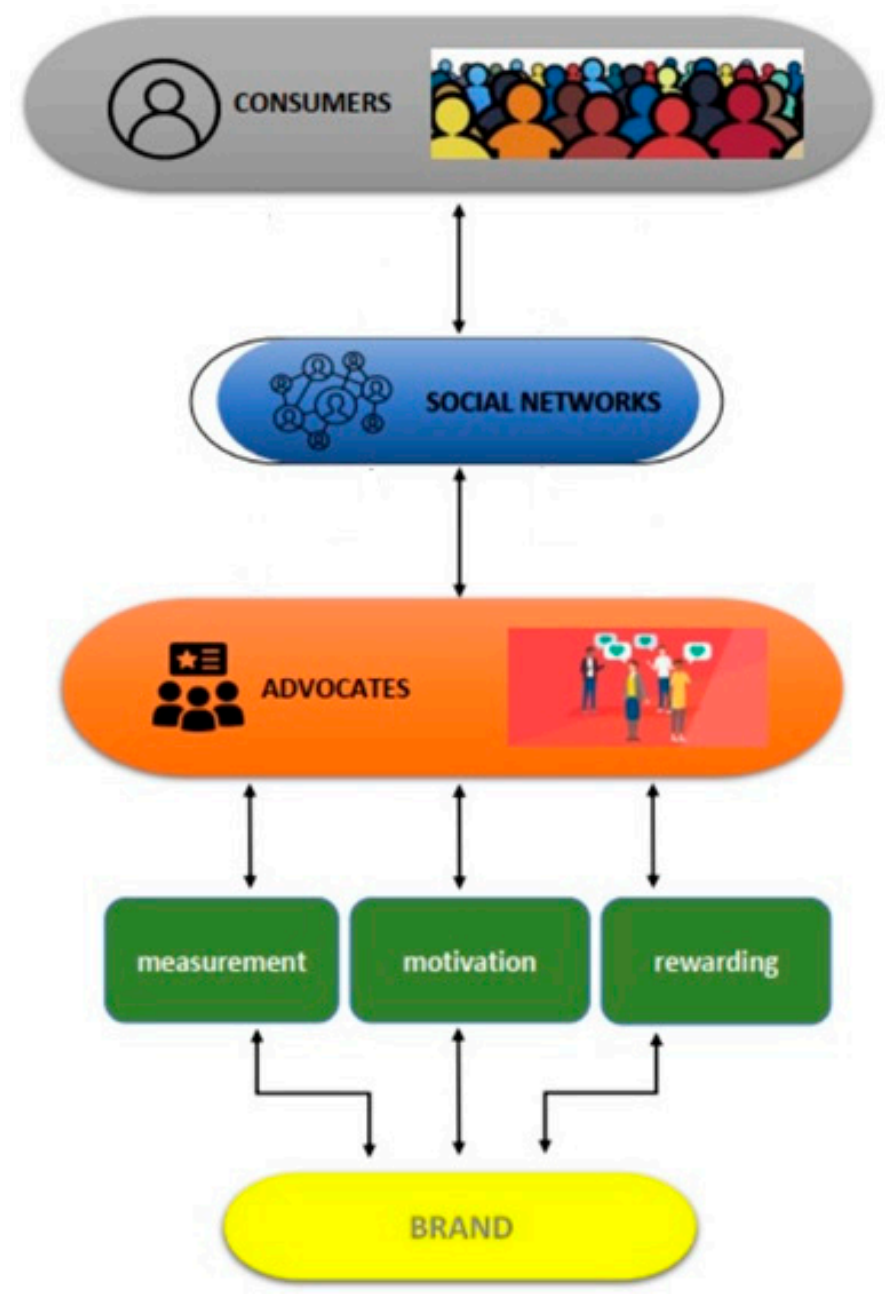

Figure 2. The architecture of the ADVO system.

\subsection{The Functions of the ADVO System}

The ADVO system has some functions to manage influencer marketing campaigns. It has the ability for data crawling on a social network automatically. Those crawled data are organized as knowledge of the brand, which are resources to determine potential influencers for marketing campaigns. Besides, the ADVO system also has the ability to produce some campaign results visually as campaign dashboards.

\subsubsection{Data Crawling}

There are three separate databases used to organize the collected data: the raw crawled database, command and mapping database, and processed database. The structures of the crawling data system are shown in Figure 3. 
The crawling system consists of four main components:

- Command receiver: the system's input is implemented as a set of external system APIs to request the crawling and when to start and end the crawling commands. A "command and mapping" database is used to save the commands for creating messages that will be sent to the message queue to process.

- Message queue: it receives and sends requests to different parts of the system. It ensures stability by using the style of asynchronous communication. This approach warrants the steadiness and performance of the system. Some popular message brokers are used, such as RabbitMQ [29], ZeroMQ [30], or streaming platforms-Kafka [31].

- Crawling worker: the core of the system, responsible for collecting the requested data. While the data collecting is sent to crawling workers by the message queue, the workers know how to obtain the needed data and which information would be saved to the "raw" database. Due to the wide variety of data sources, the system has to deal with, there are two sets of crawling workers: API requesting and user emulating. Besides, simple matching steps are used to restrict the amount of collected data. Workers will notify the "data processor" through the message queue if the desired data is saved.

- Data processor: this component pre-processes to normalize the crawled data. Those are preparation steps to make the raw data ready for external systems. This processor will remove some redundant components in raw data, such as HTML tags, and add more missing information by gathering the information from other databases. From that, the crawled data will be structured and stored in a database entirely. The results of the data processor will be saved to the processed database. At this stage, the data is considered clean and ready to be processed by external systems. External systems can consume the processed data through APIs provided by a sub-component of the crawling system or read the database directly.

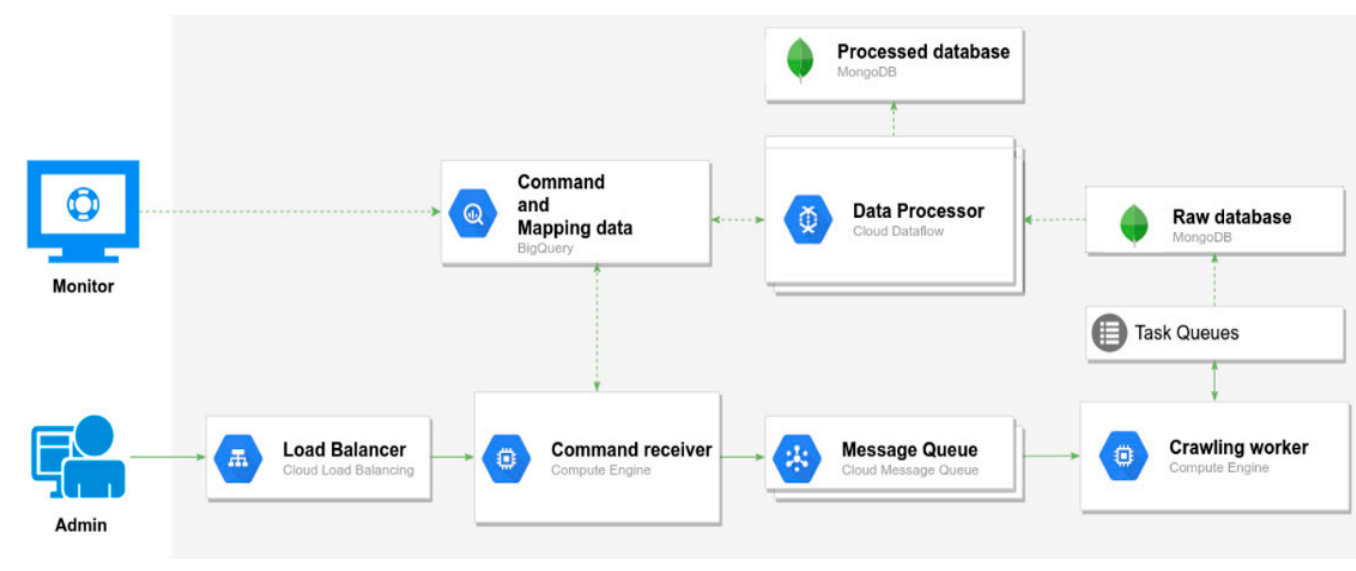

Figure 3. Data crawling system.

\subsubsection{Detecting Emerging Influencers for the Brand}

The detection of influencers is worked based on the influential measures of users for a brand on a social network. Those measures are amplification factors for evaluating the information diffusion on a social network, the passion point for quantifying the user's love of a brand, and the content creation score for determining the ability to create the post's content. They estimate the affection of a user to his/her audience. Section 4 presents the details of those measures. 
The analysis of the post's sentiment is the foundation for computing those influential measures. There are many methods for sentiment and affect analysis in the social network [32]. In order to determine the sentiment of a post, we applied zero-shot learning to feed the embedded sequence to a semantic matrix based on transfer learning [33]. The multi-task learning with the semantic matrix is used from the embedded model as inputs to classify sentiments of the post simultaneously [34]. The improvement of transformers combining the layer of Squeeze and Excitation is applied for the back-bone of the embedded model [35].

After the classification step, posts which mention the brand will undergo name entities recognition to extract brand names and technique names as candidates for matching with keywords of brands. Those keywords are created by specialists who have the best knowledge of this industry. On the other hand, to obtain more information in context, such as where the post was posted (personal wall, fan page or a group, what industries these places are?), a querying pipeline is built while the information of context from a "profiles database" is built from analyzing historical data. This module is scheduled to update the data.

Finally, all information is stored together in a pre-processed database. In fact, for this pipeline to get high accuracy, experts must verify the results and update the keywords database, testing, and re-training the model. The whole process for analyzing the sentiment of the post is shown in Figure 4.

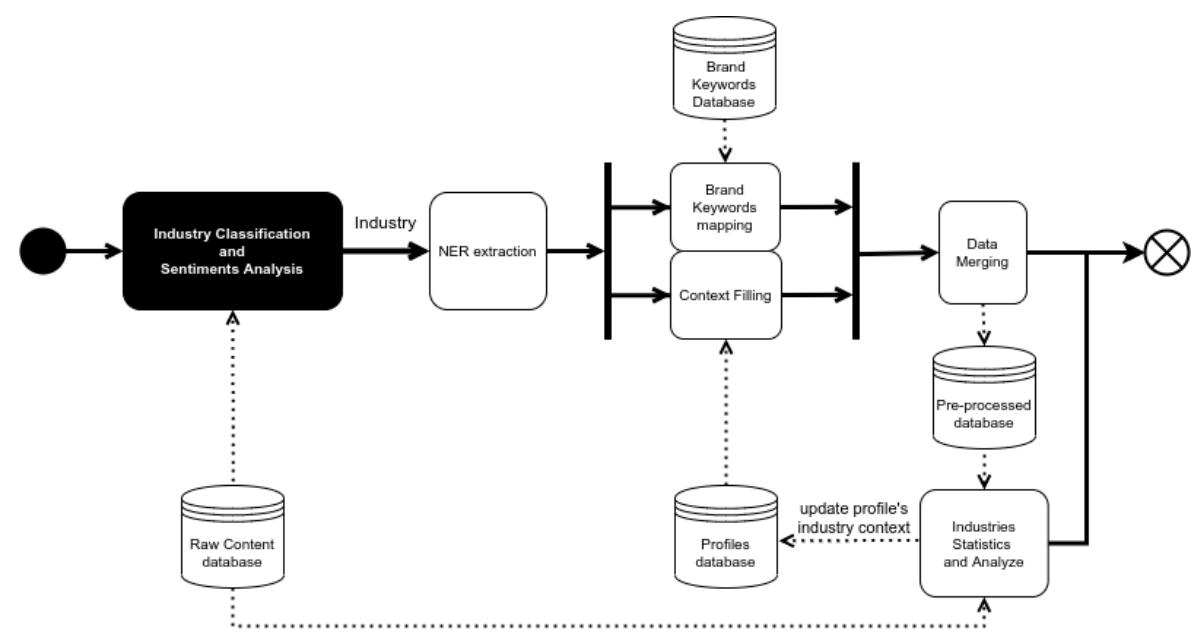

Figure 4. The process for analyzing the sentiment of posts in the ADVO system.

For a brand or a product, the management system collects information related to this brand/product. The influential measures are computed through the collected data. Those values are used to detect the list of potential influencers for the determined brand.

Figure 5 describes how the three measures' calculation is set up in our system. By extending the use of command receivers, calculation requests can be sent to the appropriate worker. The requests contain information of which score is requested, whom, and which brand/product the score points to; timing-related factors should be considered when carrying out the calculation. For the sake of single responsibility, three different sets of scoring workers are set up, each for one purpose only. 


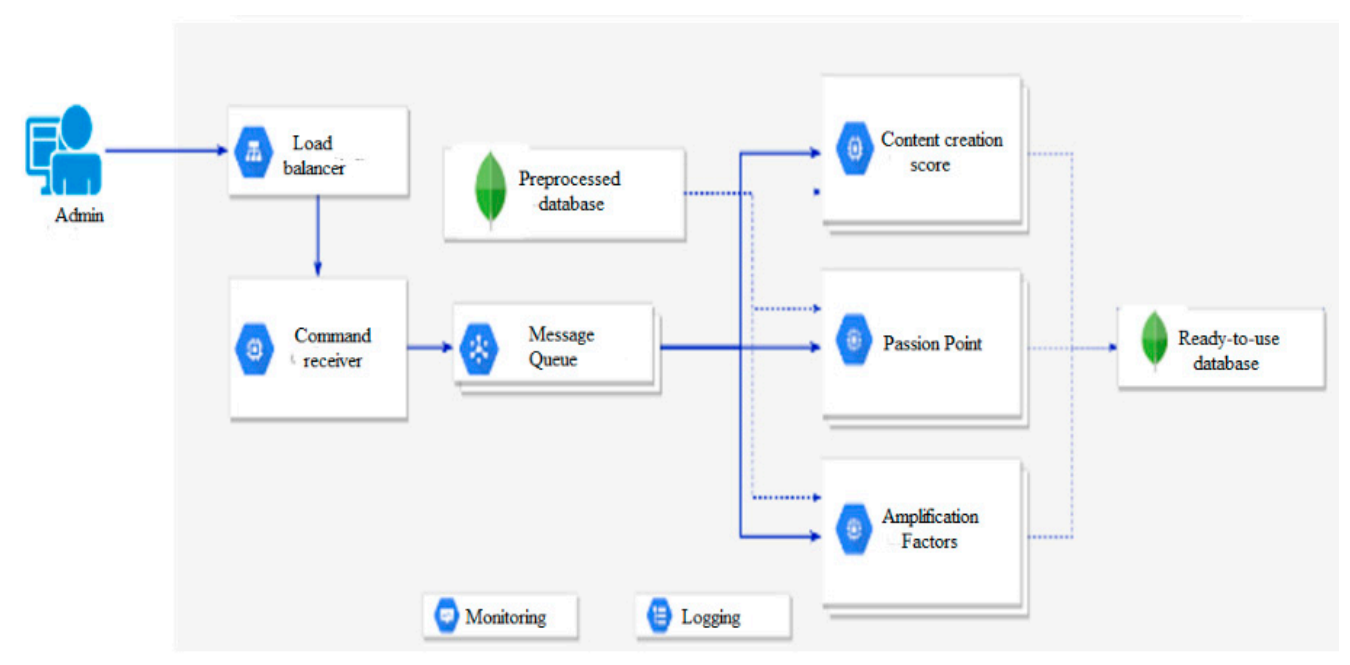

Figure 5. Determination of influential measures of users.

\subsubsection{Campaign Dashboard}

It is complicated to quantify and analyze humans based on texture and numeric data. Data, which were tracked in the marketing campaign, need to be shown in visual form. It will support to give the decision more precisely. Thus, the visualization of campaign results has to be developed as a dashboard. The dash-plot is used to build the dashboard with an interactive interface to meet business requirements. The system allows viewers to identify concerned areas on the dashboard.

In practice, with a specific campaign, businesses have different requirements for the report dashboards. To match these, our system allows administrators to customize their reports. Firstly, the system will ask which fields to be shown in the dashboard. Then, based on the data type of each field, the system will recommend some kinds of dashboards for administrators. They will choose a suitable kind or revise their requirements. The system will save those configurations for each project and show them in the future.

\section{The Measures for Detecting Influencers on Social Network}

AdvoSights is a main sub-system of the ADVO system. Based on the collected data, this sub-system helps the brands to detect potential influencers to run their influencer marketing campaigns in a duration time. This section presents a model for organizing the data on social networks, called the Social-Net model. Using this model, some parameters to estimate the impact of a user on his/her audience were proposed. Those measures are amplification factors, the passion point, and the content creation score [26,27].

In this section, we denote:

- TIME is the data type as a timestamp.

- $\operatorname{card}(\mathrm{S})$ : number of elements in S.

\subsection{Social Network}

There are many kinds of object on a social network. In this paper, two main kinds are studied: users and their posts on the network. A social network can be represented by a relationship between users and posts [26,36].

Definition 1. ([26]). A social network is a relational model, called the Social-Net model, which is represented by a triple:

$$
(\mathbf{U}, \mathbf{P}, \mathbf{A})
$$

$\mathbf{U}$ is a set of users, $\mathbf{P}$ is a set of posts, and $\mathbf{A}$ is a set of relations and actions between users and posts on the social network.

$\mathrm{U}-$ Set of users 
Each user $u \in \mathbf{U}$ has the structure as follows:

$$
u=(\text { Profile, Pos, Fr, Fol, Fold })
$$

- Profile: the personal information of a user.

- $\quad P o s=\left\{p_{u} \mid p_{u} \in \mathbf{P}\right\}$ : set of post of the user $u$.

- $F r=\{s \mid s \in \mathbf{U}$ and friend $(s, u)\}$ : the set of other users who are friends of the user $u$.

- $F o l=\{s \mid s \in \mathbf{U}$ and follow $(s, u)\}$ : the set of other users who are followers of the user $u$.

- $\quad F o l d=\{s \mid s \in \mathbf{U}$ and follow $(s, v)\}$ : the set of other users who are followed by the user $u$.

$\mathbf{P}-$ Set of posts

Each post $p \in \mathbf{P}$ is a tube as follows:

$$
p=(\text { Seeder, } \theta, \text { Content, React, Share, Comm })
$$

- $\quad$ Seeder $\in \mathbf{U}:$ the user seeds the post $p$.

- $\quad \theta \in$ Time: a timestamp when the post $p$ is posted.

- Content: the content of the post $p$.

- $\operatorname{React}=\{s \mid s \in \mathbf{U}$ and reaction $(s, p)\}$ : the set of users who have reactions with the post $p$.

- $\quad$ Share $=\{s \mid s \in \mathbf{U}$ and share $(s, p)\}$ : the set of users who share the post $p$.

- $\operatorname{Comm}=\{s \mid s \in \mathbf{U}$ and comment $(s, p)\}$ : the set of users who have comments on the post $p$.

A-Set of actions and relations

$$
\mathbf{A}=\mathbf{A}_{\mathrm{R}} \cup \mathbf{A}_{\mathrm{P}}
$$

- $\mathbf{A}_{\mathrm{R}}$ : a set of relations between two users.

$+\quad$ follower $\subseteq \mathbf{U} \times \mathbf{U}$ : the relation about following between two users.

+ $\quad$ friend $\subseteq \mathbf{U} \times \mathbf{U}$ : the relation about friendship between two users. This relation is symmetric.

- $\quad \mathbf{A}_{\mathrm{P}}$ : a set of relations as actions between a user and a post.

$+\quad$ reaction $\subseteq \mathbf{U} \times \mathbf{P} \times \mathbf{N}$ : a user reacts to a post. The kind of this reaction is a natural number.

$+\quad$ comment $\subseteq \mathbf{U} \times \mathbf{P}$ : a user comments on a post.

$+\quad$ share $\subseteq \mathbf{U} \times \mathbf{P}:$ a user shares a post.

\subsection{The Method for Detecting Influencers}

This section proposed three measures to evaluate the impact of users on a social network: Amplification factors-how far the information disseminates on a social network, Passion point-how much consumers love the brand, and Content creation-what an inspiring post looks like.

\subsubsection{The Measure of Amplification Factors}

With an influencer, besides the number of friends and followers, the interactions on each his/her post is a helpful amplification factor in evaluating the effectiveness of that user. Moreover, because a marketing campaign is only run in the duration time, the number of interactions needs to be estimated in the determined time. This section presents a method to evaluate the average of interactions in the duration time based on the social pulse $[37,38]$.

Definition 2. (Social pulse) Let (U, $\boldsymbol{P}, \boldsymbol{A})$ be a social network as Social-Net model, $u \in \boldsymbol{U}$ be a user, $p \in \boldsymbol{P}$ be a post on this social network and the time window $\delta$. 
(a) Define:

$$
\begin{array}{r}
I_{p}^{u}(\delta)=\{s \in \mathrm{U} \mid s \neq u, s \in(p \cdot \text { React } \cup p \cdot \operatorname{Sh} \cup p . \text { Comm }) \\
\text { in the time } \in[p \cdot \theta, p \cdot \theta+\delta]\}
\end{array}
$$

It means: $I_{p}{ }^{u}(\delta)$ is the set of users who interacted with the post $p$ in the duration time $\delta$ from the moment that $p$ was posted, $p . \theta$

(b) The social pulse is the total interactions with the post $p$ in the time window $\delta$. It is computed by:

$$
\operatorname{SoP}_{p}(\delta)=\sum_{v \text { shared post } p} \operatorname{card}\left(I_{p}^{v}(\delta)\right)
$$

Definition 3. (Average of Interactions) Given a social network $(\boldsymbol{U}, \boldsymbol{P}, \boldsymbol{A})$ as Social-Net model, and $a$ user $u \in U$. The average of interactions for each post of the user $u$ in the time window $\delta$ is determined by:

$$
A v I_{u}(\delta)=\frac{\sum_{p \in u . P o s} \operatorname{SoP}_{p}(\delta)}{\operatorname{card}(u \cdot P o s)}
$$

\subsubsection{Passion Point}

If a user loves a brand $X$, he/she will create positive posts for it. The user also usually propagates the information linked to brand $X$. The passion point computes the fondness of a user with a determined brand. It belongs to the distribution of positive posts and the frequency of appearance of those posts.

Section 3.2 presented the process for analyzing the sentiment of posts of the ADVO system (Figure 4). This process is worked to enhance the self-attention network by combining with squeeze and excitation layers [34,39]. The distribution of positive posts is a normal, binomial distribution [40], and the binomial proportion confidence interval can be determined based on the Wilson score interval method. Thus, the passion point can be computed by using the Wilson score.

Definition 4. ([27]). (Passion point) Let $u \in U$ be a user, the brand $X$ on a social network as Social-Net model.

(a) The ranking score for the user $u$ with the brand $X$ :

$$
\operatorname{ranking}_{X}(u):=\frac{\rho+\frac{z^{2}}{2 \cdot \operatorname{card}\left(P_{u}\right)}}{1+\frac{z^{2}}{\operatorname{card}\left(P_{u}\right)}}-\frac{z}{1+\frac{z^{2}}{\operatorname{card}\left(P_{u}\right)}} \sqrt{\frac{\rho(1-\rho)}{\operatorname{card}\left(P_{u}\right)}+\frac{z^{2}}{4\left(\operatorname{card}\left(P_{u}\right)\right)^{2}}}
$$

where, $\rho=\operatorname{card}\left(\operatorname{Pos}_{u}^{\text {positive }}\right) / \operatorname{card}($ u.Pos): the binomial proportion.

$\operatorname{Pos}_{u}^{\text {positive }}$ is the set of positive posts of the user $u$ with the brand $X$.

$z$ : the quantile of a standard normal distribution.

(b) The formula for computing of passion point of the user $u$ with the brand $X$ is:

$$
P P_{X}(u):=\frac{\operatorname{card}\left(P_{u}^{\text {positive }}\right)}{n_{\text {day }}} \cdot\left(\operatorname{ranking}_{X}(u)+\log (u . P o s)\right)
$$

where $n_{\text {day }}=$ the number of report days.

\subsubsection{Content Creation Score}

A product creates content to boost the purchase of consumers. The content is the bridge between the product and its customers. Hence, the attraction by insight posts will make it more attractive to their customers, mainly targeted customers. Thus, the ability to 
create posts well is a useful parameter to measure the influence of a user, called content creation score.

In practice, some posts gain lots of interactions from the audience, although they are meaningless because of the authority of their seeders. In this study, a meaningless post is a post in which the number of its words is smaller than the average of words in each post [27]. The content creation score of a user is only estimated based on meaningful posts. Given a post $p$, denote:

- $\quad \operatorname{word}(p)$ : the number of words in the post $p$.

- $w_{\text {positve }}(p)$ : the number of positive words in the post $p$.

Definition 5. ([27]). (Quality of posts) Given a social network (U, P, A) as Social-Net model, and a user $u \in \boldsymbol{U}$ on this social network.

(a) The average of words in each post of the user $u$ :

$$
\omega:=\frac{\sum_{p \in u . P o s} \operatorname{word}(p)}{\operatorname{card}(\text { u.Pos })}
$$

(b) The quality of posts of the user $u, Q(u)$, is computed by:

$$
\begin{aligned}
& \sum_{p_{u} \in \text { u.Pos }} \frac{\text { word }_{\text {positve }}\left(p_{u}\right)}{\text { word }\left(p_{u}\right)} \\
& \operatorname{Quality}(u):=\frac{\operatorname{word}\left(p_{u}\right) \geq \omega}{\operatorname{card}\left(\left\{p_{u} \in u . \operatorname{Pos} \mid \operatorname{word}\left(p_{u}\right) \geq \omega\right\}\right)}
\end{aligned}
$$

Definition 6. (Content creation score) Let $(\boldsymbol{U}, \boldsymbol{P}, \boldsymbol{A})$ be a social network as Social-Net model, $u \in$ $U$ be a user, and the brand $X$. The quantitation of the content creation score of the user $u$ for the brand $X$, called Cont $_{X}(u)$, is as follows:

$$
\operatorname{Cont}_{X}(u):=\log (Q u a l i t y(u))+P P_{X}(u)
$$

where, $P P_{X}(u)$ and $Q u a l i t y(u)$ are computed by the Formulas (5) and (7), resp.

\section{Testing and Experimental Results}

Fashion is an attractive industry. There are many shopping seasons for fashion in a year, such as fashion weeks. The ADVO system was used to manage a practical influencer marketing campaign from 24 February-12 March 2020. The customer of our system is a fashionable brand in Vietnam so the marketing campaign only considers Vietnamese users on Facebook.

\subsection{Dataset}

As the amount of data is huge and its structure varies from source to source, MongoDB, a popular No-SQL database, is our choice to save the raw data [41]. On the other hand, the preprocessed database is based on MongoDB too, but it can be based on any related database system if needed. Figure 6 describes the raw database with the connection between tables that store crawled data. Figure 7 shows the preprocessed data. 


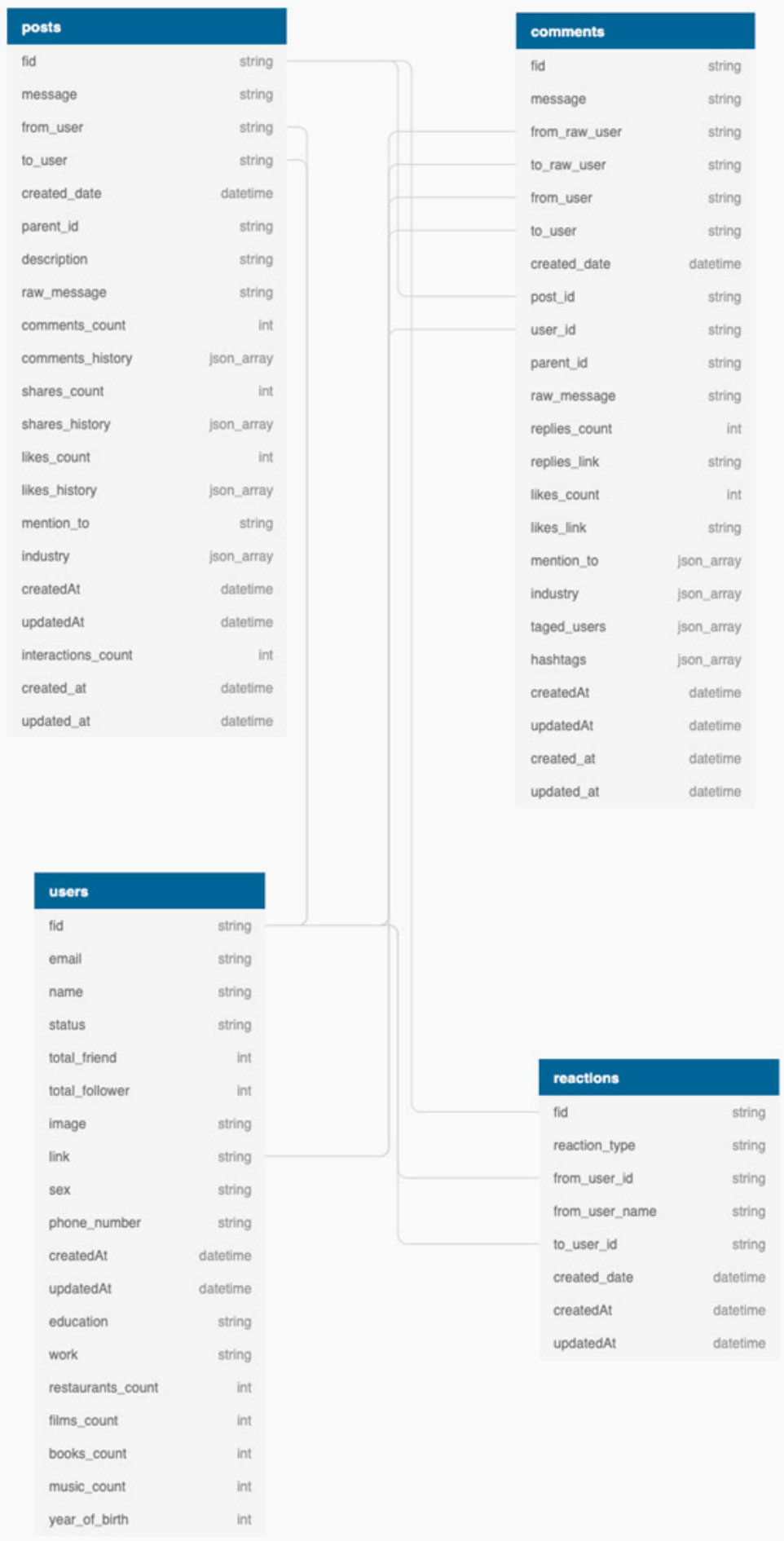

Figure 6. Raw database schema. 


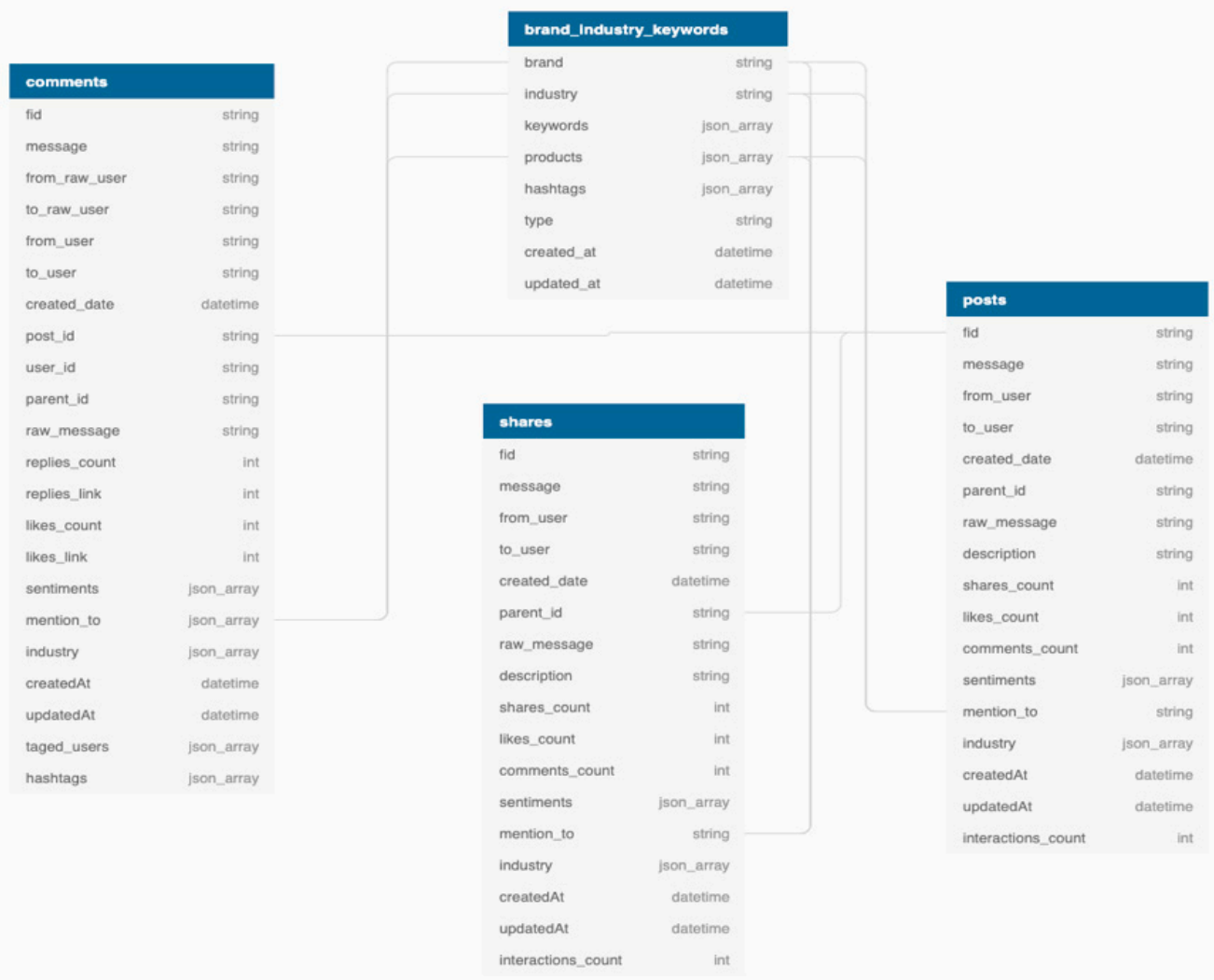

Figure 7. Preprocessed database schema.

A list of 700 social network users who are potential influencers in the fashion industry is collected based on their activities on fashion-related groups/pages and their fashionrelated social content. Using the crawling system, the users' activity data are recorded from June to December 2019. The dataset of this phase is crawled on Facebook from Vietnamese users who are working in fashion. Those are used for influencer determination.

Table 1 presents the raw data that has been crawled, and the resulted data remained after preprocessing and filtering.

Table 1. The data collected from June to December 2019 in fashion.

\begin{tabular}{ccccc}
\hline Database & Users & Posts & Comments & Reactions \\
\hline Raw & 700 & 172,028 & $1,897,009$ & $12,093,796$ \\
Preprocessed & 700 & 1472 & 10,039 & 139,012 \\
\hline
\end{tabular}

\subsection{The Practical Influencer Marketing Campaign}

For testing the ADVO system, when the brand ran its influencer marketing campaign from 24 February-12 March 2020, there were two phases as follows:

- $\quad$ Phase 1: The customer identified 13 micro-influencers. The sales department of our customers determined those influencers. This phase was run from 24 February-1 March 2020.

- Phase 2: The customer applied AdvoSights of the ADVO system to detect 10 microinfluencers using the measures in Section 4. This phase was run from 7-12 March 2020.

Using the collected data, the influential measures in Section 4 of each user were computed and used to detect influencers of the brand. Later, the customer managed its campaign through AdvoBiz of the ADVO system. When running a marketing campaign, each micro-influencer will generated their own link by AdvoBiz and they will post their links in the post. Hence, when audience click or order through those links, the obtained 
revenue will be computed for the corresponding link owner. The ADVO system can manage all the interaction processes with the link of an influencer, such as interaction (reaction, comment, share), click on link and order products. Those processes are undertaken in AdvoBiz. It checks and statistics the number of interactions or recommendations between users on its environment. Thus, this helps influencers only focus to attract their audience and click on their order link through their interesting posts. The results of this campaign are shown in the next section.

\subsection{Experimental Results}

The practical sales campaign was carried out in the marketing campaign to experiment ADVO system. The profiles of social influence and sales outcome were recorded to compare the effectiveness of two phases in the campaign. The total of interactions on the brand is shown in Figure 8. In those results, the posts only count for influencers. Table 2 gives the details of interactions for each phase.

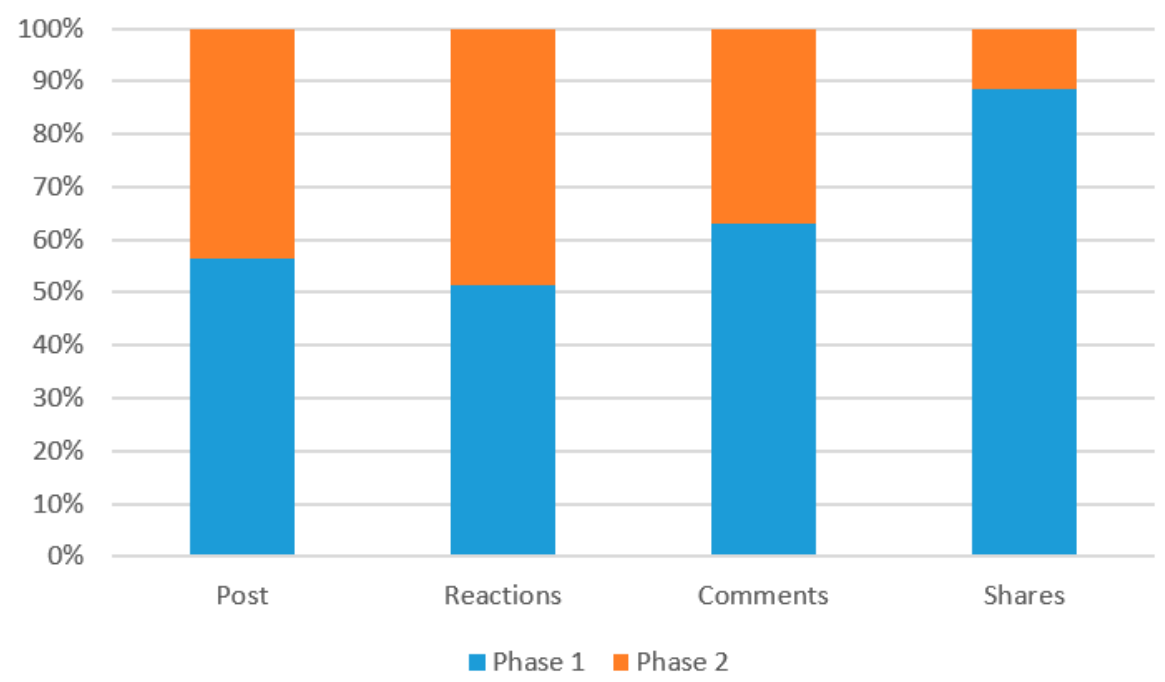

Figure 8. The interactions rate between two phases in the influencer marketing campaign from 24 February-12 March 2020.

Table 2. Total of interactions for each phase.

\begin{tabular}{cccccc}
\hline $\begin{array}{c}\text { Type of } \\
\text { Interactions }\end{array}$ & Posts & Reactions & Comments & Shares & $\begin{array}{c}\text { Total of } \\
\text { Interactions }\end{array}$ \\
\hline Phase 1 & 13 & 5139 & 869 & 390 & $\mathbf{6 3 9 8}$ \\
Phase 2 & 10 & 4849 & 509 & 51 & $\mathbf{5 4 0 9}$ \\
\hline Total & $\mathbf{2 3}$ & $\mathbf{9 9 8 8}$ & $\mathbf{1 3 7 8}$ & $\mathbf{4 4 1}$ & $\mathbf{1 1 , 8 0 7}$ \\
\hline
\end{tabular}

Table 3 analyzes the sentiment of comments on posts of influencers. Those comments are concerned with the product in two phases.

Table 3. Sentiments of posts and comments in two phases.

\begin{tabular}{cccccc}
\hline Phase & Positive & Negative & Neutral & Not concern & Total \\
\hline Phase 1 & 337 & 7 & 93 & 432 & 869 \\
Phase 2 & 281 & 4 & 83 & 141 & 509 \\
\hline
\end{tabular}

The rate of "not concerned" comments and total comments in phase 1 is (49\%). It is near double the rate of that in Phase $2(27 \%)$. Influencers attracted the audience of influencers in phase 1; they were not attracted to the brand. However, the audience of 
influencers in phase 2 was attracted by the brand mentioned by their influencers. Besides, the rate of positive comments in phase 2 was also higher than in phase 1.

The rate of click per interaction reflects the attraction of a post to the audience. The conversion rate for each click in a marketing campaign is required to measure the conversion from interesting to consumers' purchase decisions. Hence, those rates are essential to evaluate the effectiveness of a marketing campaign. Table 4 shows their values in our customers' campaign from 24 February-12 March 2020.

Table 4. The rates for each interaction and click.

\begin{tabular}{ccc}
\hline & Phase $\mathbf{1}$ & Phase 2 \\
\hline Interactions & 6398 & 5409 \\
Clicks & 1428 & 817 \\
Orders & 157 & 188 \\
Rate of click per interaction & $\mathbf{2 2 . 3 2 \%}$ & $\mathbf{1 5 . 1 \%}$ \\
Conversion rate per click & $\mathbf{1 0 . 9 9 \%}$ & $\mathbf{2 3 . 0 1 \%}$ \\
\hline
\end{tabular}

Although the total clicks in phase 2 are lower than in phase 1 , the conversion rate per click of phase 2 is better than in phase 1 . In phase 1, the audience may be clicked to the link to the product by the authority of influencers, and they are not ready to buy the product. In phase 2 , the audience is interested in the product through the attraction of the influencers. Hence, when they click on the link to a product, they want to reserve it.

Besides, the conversion rate of phase 2 displays that the revenue of this phase is better than another. The average influencer efficiency of phase 1 is also lower than phase 2 . This is shown as Figure 9.

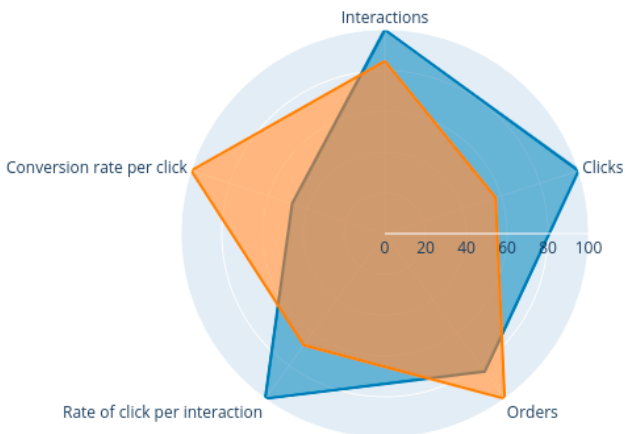

a. The efficiency for all influencers in each phase.

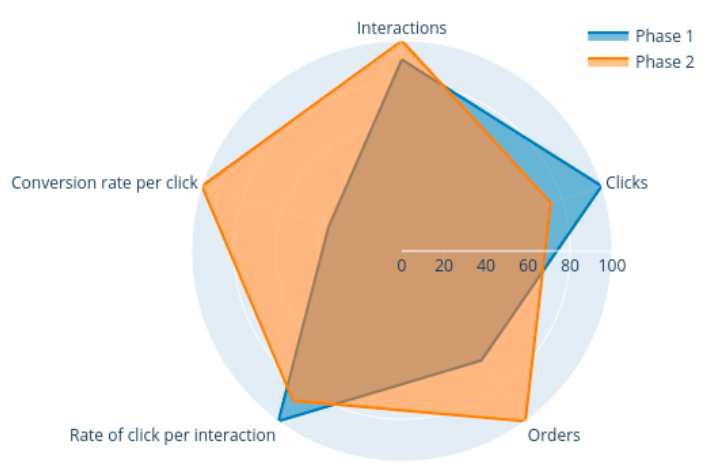

b. Average efficiency for each influencer in each phase.

Figure 9. The average influencer efficiency.

\section{Discussion}

Nowadays, there are many systems to detect influencers as well as manage influencer marketing campaigns. Hiip is an influencer platform in Southeast Asia [23]. It helps brands connecting with targeted influencers categorized into segments with fanbases. However, it only detects influencers through their interaction and does not mention their content creation. Traackr is the record system for data-driven influencer that marketers use to invest the right strategies, streamline campaigns, and scale programs [42]. The criteria for determining influencers in this system are their suitability for brands, the insight from customers, demographics, and love of the brand.

Nonetheless, this system does not support managing a marketing campaign. ViralWorks is an influencer platform for marketing based on the intensive network powered by artificial intelligence technology [43]. This system helps the brands to create viral social media through influencers who have signed up for this platform. However, the data of 
this system is only built based on its users and brands which were signed up; thus, this decreases the appropriate data in practice.

The influencers detected by the AVDO system can turn interactions into revenue effectively, because those influencers approach more targeted customers. The ADVO system gives visual reports of the marketing campaign as dash-boards. It performs users' work on each influencer's posts and the revenue each influencer receives. Moreover, the consumer behaviors can be traced when they interact with influencers. Those help the brand to build its homophily which is the brand lovers' community.

\section{Conclusions and Future Works}

This study proposed the architecture of a management system for influencer marketing campaigns on a social network. This system is called the ADVO system. The proposed architecture includes three sub-systems: AdvoSights-this system helps estimate the behaviors of users on a social network for detecting potential influencers, AdvoBiz-this is the tool for the entrepreneurs to deploy their commercial activities, including the management of marketing campaigns, and AdvoFair - this builds an advocate community of the brand.

The ADVO system is a foundation supporting businesses to manage their commercial activities through an influencer marketing campaign. Besides extract visual reports for the marketing campaign, the system can detect suitable influencers for a brand. The ADVO system has been tested and achieved positive experimental results from customers in real-world influencer marketing campaigns.

However, the current ADVO system only can work for a small marketing campaign in a local market. This system will be augmented to manage the more extensive campaigns with many users and a higher frequency of interactions for the duration of the campaigns. Some methods for increasing the attraction and influence of users will also be researched, such as using reinforcement learning methods [44] to improve the content creation of users. Those methods would escalate the usefulness of influencers in the marketing campaign. The recognition of consumer behaviors is vital to approach target customers. In further research, through the ADVO system, our customers can track the activities of consumers on the social network and establish useful plans of marketing campaigns.

Moreover, there are many other media platforms of social networks; our method will be tested on other platforms of social networks, such as images (Instagram [45]), and videos (Tiktok [46]). Hence, the AVDO system needs to be improved to manage influencer marketing campaigns on those media platforms.

Author Contributions: Conceptualization, T.H., H.D.N. and I.Z.; methodology, T.H., H.D.N. and I.Z.; software, K.V.N. and V.T.P.; resources, K.V.N. and S.N.H.; writing-original draft preparation, T.H., H.D.N., S.N.H. and V.T.P.; writing-review and editing, I.Z. and K.V.N.; visualization, K.V.N., S.N.H. and V.T.P. All authors have read and agreed to the published version of the manuscript.

Funding: This work was funded by Gia Lam Urban Development and Investment Company Limited, Vingroup and supported by Vingroup Innovation Foundation (VINIF) under project code DA132-15062019.

Conflicts of Interest: The authors declare no conflict of interest.

\section{References}

1. Deiss, R.; Henneberry, R. Digital Marketing for Dummies; Wiley: Hoboken, NJ, USA, 2016.

2. Dey, N.; Borah, S.; Babo, R.; Ashour, A. Social Network Analytics: Computational Research Methods and Techniques; Academic Press: Cambridge, MA, USA, 2018.

3. Tabellion, J.; Esch, F. Influencer Marketing and its Impact on the Advertised Brand. In Advances in Advertising Research X; Bigne, E., Rosengren, S., Eds.; Springer Nature: Berlin/Heidelberg, Germany, 2019; pp. 29-41.

4. Vo, L. Mining Social Media_Finding Stories in Internet Data; No Starch Press: San Francisco, CA, USA, 2019.

5. Levin, A. Influencer Marketing for Brands; Springer Nature: Berlin/Heidelberg, Germany, 2019.

6. Zimmerman, J.; Ng, D. Social Media Marketing All-in-One; Wiley: Hoboken, NJ, USA, 2017.

7. Laroche, M.; Habibi, M.; Richard, M.; Sankaranarayanan, R. The effects of social media based brand communities on brand community markers, value creation practices, brand trust, and brand loyalty. Comput. Hum. Behav. 2012, 28, 1755-1767. [CrossRef] 
8. Mediakix. Influencer Marketing 2019-Key Statistics from Our Influencer Marketing Survey. Available online: https://mediakix. com/influencer-marketing-resources/influencer-marketing-industry-statistics-survey-benchmarks/ (accessed on 3 June 2021).

9. Chen, W.; Lakshmanan, L.; Castillo, C. Information and Influence Propagation in Social Networks. Synth. Lect. Data Manag. 2013, 5, 1-177. [CrossRef]

10. Sun, Y.; Yin, H.; Wen, J.; Sun, Z. Urban Region Function Mining Service Based on Social Media Text Analysis. Int. J. Softw. Eng. Knowl. Eng. 2021, 31, 563-586. [CrossRef]

11. García, A.; Gil-Mediavilla, M.; Álvarez, I.; Casares, M. The Influence of Social Networks within Educational and Social Fields: A Comparative Study between Two Generations of Online Students. Sustainability 2020, 12, 9941. [CrossRef]

12. Lee, M.; Oh, S. An Information Recommendation Technique Based on Influence and Activeness of Users in Social Networks. Appl. Sci. 2021, 11, 2530. [CrossRef]

13. Do, N.V.; Nguyen, H.; Selamat, A. Knowledge-Based Model of Expert Systems Using Rela-Model. Int. J. Softw. Eng. Knowl. Eng. 2018, 28, 1047-1090. [CrossRef]

14. Wagih, H.M.; Mokhtar, H.M.O.; Ghoniemy, S.S. Exploring Trusted Relations among Virtual Interactions in Social Networks for Detecting Influence Diffusion. ISPRS Int. J. Geo-Inf. 2019, 8, 415. [CrossRef]

15. Nguyen, H.D.; Pham, V.T.; Le, T.T.; Tran, D.H. A Mathematical Approach for Representation Knowledge about Relations and Its Application. In Proceedings of the 2015 IEEE International Conference on Knowledge and Systems Engineering (KSE 2015), Ho Chi Minh City, Vietnam, 8-10 October 2015; pp. 324-327.

16. Cvijikj, I.P.; Michahelles, F. Online engagement factors on Facebook brand pages. Soc. Netw. Anal. Min. 2013, 3, 843-861. [CrossRef]

17. López, L.M. Variables of twitter's brand activity that influence audience spreading behavior of branded content. ESIC Mark. Econ. Bus. J. 2018, 44, 525-546.

18. Riquelme, F.; González-Cantergiani, P. Measuring user influence on Twitter: A survey. Inf. Process. Manag. 2016, 52, 949-975. [CrossRef]

19. Huynh, T.; Zelinka, I.; Pham, H.; Nguyen, H. Some influence measures to detect the influencer on social network based on Information Propagation. In Proceedings of the 9th International Conference on Web Intelligence, Mining and Semantics (WIMS 2019), Seoul, Korea, 26-29 June 2019.

20. Tidke, B.; Mehta, R.; Dhanani, J. Consensus-based aggregation for identification and ranking of top-k influential nodes. Neural Comput. Appl. 2020, 32, 10275-10301. [CrossRef]

21. Qiu, L.; Zhang, S.; Gu, C.; Tian, X. Scalable Infuence Maximization Meets Efficiency and Effectiveness in Large-Scale Social Networks. Int. J. Soft Eng. Knowl. Eng. 2020, 30, 1079-1096. [CrossRef]

22. Taheri, S.; Bouyer, A. Community Detection in Social Networks Using Affinity Propagation with Adaptive Similarity Matrix. Big Data 2020, 8, 189-202. [CrossRef] [PubMed]

23. Hiip. Available online: https:/ / hiip.asia/influencer/ (accessed on 3 June 2021).

24. 7Saturday. Available online: https://7saturday.com/en/index.html (accessed on 3 June 2021).

25. Activate. Available online: https:/ / try.activate.social (accessed on 3 June 2021).

26. Huynh, T.; Nguyen, H.; Zelinka, I.; Dinh, D.; Pham, X.H. Detecting the Influencer on Social Networks Using Passion Point and Measures of Information Propagation. Sustainability 2020, 12, 3064. [CrossRef]

27. Nguyen, H.D.; Huynh, T.; Luu, S.; Hoang, S.N.; Pham, V.T.; Zelinka, I. Measure of the content creation score on social network using sentiment score and passion point. In Proceedings of the 19th International Conference on Intelligent Software Methodologies, Tools, and Techniques (SOMET 2020), Kitakyushu, Japan, 22 September 2020; pp. 425-434.

28. Valdiviezo, O.; Sánchez, J.; Cervantes, O. Visualizing sentiment change in social networks. In Proceedings of the 8th Latin American Conference on Human-Computer Interaction, Antigua Guatemala, Guatemala, 8-10 November 2017.

29. RabbitMQ. Available online: https:/ / www.rabbitmq.com/ (accessed on 3 June 2021).

30. ZeroMQ. Available online: https:/ / zeromq.org/ (accessed on 3 June 2021).

31. Kafka. Available online: https:/ / kafka.apache.org/ (accessed on 3 June 2021).

32. Patil, H.; Atique, M. CDNB: CAVIAR-Dragonfly Optimization with Naive Bayes for the Sentiment and Affect Analysis in Social Media. Big Data 2020, 8, 107-124. [CrossRef] [PubMed]

33. Tao, J.; Fang, X. Toward multi-label sentiment analysis: A transfer learning based approach. J. Big Data 2020, 7, 1-26. [CrossRef]

34. Nguyen, H.; Huynh, T.; Hoang, S.; Pham, V.; Zelinka, I. Language-oriented Sentiment Analysis based on the grammar structure and improved Self-attention network. In Proceedings of the 15th International Conference on Evaluation of Novel Approaches to Software Engineering (ENASE 2020), Prague, Czech Republic, 5-6 May 2020; pp. 339-346.

35. Hu, J.; Shen, L.; Sun, G. Squeeze-and-Excitation Networks. In Proceedings of the Conference on Computer Vision and Pattern Recognition (CVPR 2018), Salt Lake City, UT, USA, 18-22 June 2018.

36. Do, N.V.; Nguyen, H.D.; Mai, T.T. A Method of Ontology Integration for Designing Intelligent Problem Solvers. Appl. Sci. 2019, 9 , 3793. [CrossRef]

37. Pham, H.; Jung, J.; Hwang, D. Beating Social Pulse: Understanding Information Propagation via Online Social Tagging Systems. J. Univers. Comput. Sci. 2012, 18, 1022-1031. [CrossRef] 
38. Nguyen, H.; Nguyen, K.; Hoang, S.; Huynh, T. Design a management system for the influencer marketing campaign on social network. In Proceedings of the 9th International Conference on Computational Data and Social Networks (CSoNet 2020), Dallas, TX, USA, 11-13 December 2020; pp. 139-151.

39. Nguyen, H.D.; Le, T.; Tran, K.; Luu, S.; Hoang, S.; Phan, H. Multi-level Sentiment Analysis of Product Reviews based on Grammar Rules of Language. In Proceedings of the 19th International Conference on Intelligent Software Methodologies, Tools, and Techniques (SOMET 2021), Cancún, Mexico, 21-23 September 2021; in press.

40. Kaas, R.; Buhrman, J. Mean, Median and Mode in Binomial Distributions. Stat. Neerl. 1980, 34, 13-18. [CrossRef]

41. MongoDB. Available online: https:/ / www.mongodb.com/cloud/atlas (accessed on 3 June 2021).

42. Traackr. Available online: https:/ / www.traackr.com/ (accessed on 29 June 2021).

43. ViralWorks. Available online: https://viralworks.com (accessed on 29 June 2021).

44. Huynh, A.T.; Nguyen, B.T.; Nguyen, H.T.; Vu, S.; Nguyen, H. A method of Deep Reinforcement Learning for Simulated Autonomous Vehicle Control. In Proceedings of 16th International Conference on Evaluation of Novel Approaches to Software Engineering (ENASE 2021), online, 26-27 April 2021; pp. 372-379.

45. Instagram. Available online: https:/ / www.instagram.com/ (accessed on 3 June 2021).

46. Tiktok. Available online: https:/ / www.tiktok.com/en/ (accessed on 3 June 2021). 